\title{
Skull Base Giant Cell Tumors - A Case Series and Review of Literature
}

\author{
Sibhi Ganapathy ${ }^{1 *}$ and Vidya Baliga ${ }^{2}$ \\ ${ }^{1}$ Associate Consultant, Department of Neurosurgery, Manipal Hospital Whitefield, \\ Bangalore, India \\ ${ }^{2}$ Consultant Pathologist, Department of Pathology, Manipal Hospital Whitefield, \\ Bangalore, India \\ *Corresponding Author: Sibhi Ganapathy, Associate Consultant, Department of \\ Neurosurgery, Manipal Hospital Whitefield, Bangalore, India.
}

Received: March 18, 2020

Published: March 30, 2020

(C) All rights are reserved by Sibhi

Ganapathy and Vidya Baliga.

\begin{abstract}
Introduction: Skull Base Giant Cell Tumors (GCT) are rare tumors of bone usually seen in the epiphyseal edges of long bones. They are common in young adults and have a slight female preponderance. Their Axial skeleton incidence is very rare, which makes their identification and management challenging. Although surgery is the main modality of management, the presence of sensitive structures in the vicinity makes for the necessity of multi-modality therapy to allow acceptable disease free and symptom free survival. Case Report: We present two different patients with skull base GCT. The first is a central skull base GCT with two recurrences due to inadequate therapy. The challenge in this patient was diagnosis and management of the recurrent tumor without worsening the neurological deficits. New age Chemotherapy along with a judicious use of biopsy resulted in an excellent long-term response.

The second patient had a dumbbell shaped middle cranial fossa mass on the cavernous sinus extending from the orbital apex to the posterior cranial fossa. The histopathological diagnosis came as a surprise as all radiological and operative inputs pointed to a trigeminal schwannoma. This report highlights the need to keep an open mind and suspect a GCT even in improbable scenarios.

Conclusion: Skull base GCTs are rare but complex tumors owing to their low incidence and complex anatomical challenges presented by the location and behavior of the lesions. A high index of suspicion along with thorough knowledge of anatomy of the skull base is essential for management of these tumors. Despite surgery being the preferred modality of treatment, complex anatomical predicaments require adjuvant therapies to be employed to effect disease free and symptom free survival. A multidisciplinary approach is recommended for ensuring the best outcomes for patients.
\end{abstract}

Keywords: Giant Cell Tumor; Skull Base; Chemotherapy

\section{Introduction}

Giant cell tumor of bone (GCT; or osteoclastoma) is an uncommon, benign, osteolytic neoplasm. GCT comprises 3 to $5 \%$ of all primary bone tumors and occurs predominantly in early adulthood (peak incidence ages, 20 - 40 years) with a slight female predominance (3:2) [1-3]. These tumors are thought to originate from neoplastic non-osteogenic stromal cells of the bone marrow and are characterized histologically by numerous multinucleated osteoclastic giant cells diffusely distributed among a background of mononuclear stromal and macrophage lineage cells [1-3].

Although involvement of the appendicular skeleton is more typical for GCT, axial skeleton involvement, especially of the cranial and facial bones, has also been reported and is becoming increa- singly appreciated in the literature. Approximately $2 \%$ of GCTs involve the head and neck [4-6]. Involvement of the axial skeleton is often associated with increased morbidity because of local infiltration of critical structures and the associated difficulty of complete tumor resection, particularly compared with resection of GCTs of the appendicular skeleton [8]. GCT of the skull has also been reported to behave in a locally aggressive fashion.

\section{Case Report \\ Case Report 1}

A 26 year male patient presented with progressive headaches worsening for the last 6 months with on and off double vision. He was evaluated with CT and MRI, which, showed soft tissue density lesion inside the sphenoid sinus extending into the Sella and central skull base (Figure $1 \mathrm{a}$ and $1 \mathrm{~b}$, figure 2 ). 


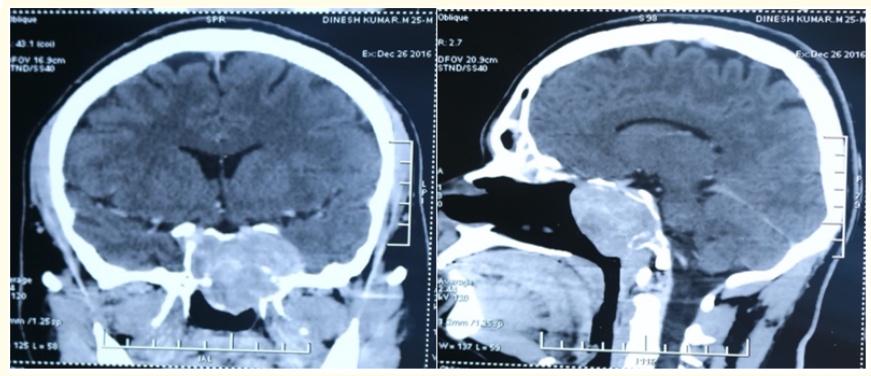

Figure 1a and 1b: Coronal \& Sagittal CT scan showing the tumor in the central Skull Base extending predominantly to the left side, in the suprasellar region extending into the cervical spine.
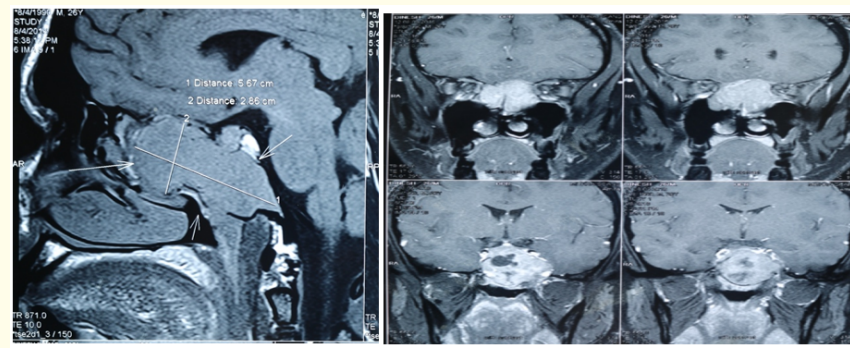

Figure 2: Showing different coronal contrast and sagittal slices of a T1 images of the brain showing the tumor' extent and its association with the carotids and cavernous sinuses.

He underwent endoscopic trans nasal biopsy done, which was inconclusive. The decompression was insufficient and hence his symptoms persisted. He was subjected to another trans nasal biopsy 6 weeks later. At this time, the tumor was substantially decompressed under Neuronavigation guidance. After surgery however, his double vision, worsened significantly. The double vision was associated with numbness of the face and lips on the right side which progressively worsened. No adjuvant therapy was given despite the histopathological diagnosis being known, and the patient was lost to follow up (Figure 3a-3c).

2 months later he again presented to a different hospital with worsened diplopia and facial numbness along with unilateral ophthalmoplegia. Imaging was done again which showed a regrowth of the lesion.

Histopathology showed a Biphasic tumor with fibrillary stroma with ganglionic differentiation and interspersed multiple foci of small cells (embryonal) with hyperchromatic nuclei, scant cytoplasm, brisk mitosis and necrosis with apoptotic bodies. The cells showed perivascular resetting occasional focus of palisading cells and papilla like configuration. In Immunochemistry, INI-1 was retained, Neu-N was positive, Synaptophysin was positive in the

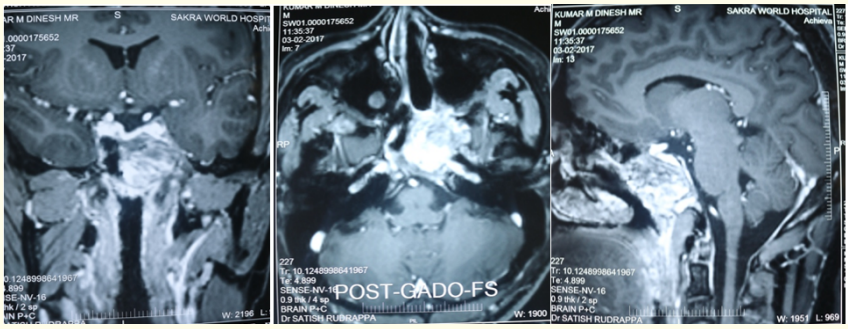

Figure 3a and 3b: Shows condition of the tumor when seen at the tertiary centre post surgery. MRI contrast images in coronal, axial and sagittal planes show some decompression but significant tumor regrowth.

fibrillary stroma and negative in the embryonal component, GFAP was negative and the MIB index was greater than $80 \%$ showing a very high grade GCT.

He was thus, counselled and sent for neo adjuvant chemotherapy. Denosuzimab (Zoledronic acid) was given along with calcium gluconate and Vitamin D3 supplementation. The therapy was given once a week for 6 months. To assist in tumor reduction and to slow growth, tumor feeder embolization was done under angiographic guidance. His tumor significantly reduced to less than $50 \%$ of its pre-chemotherapy size with resolution of diplopia and ophthalmoplegia (Figure 4a-4c).
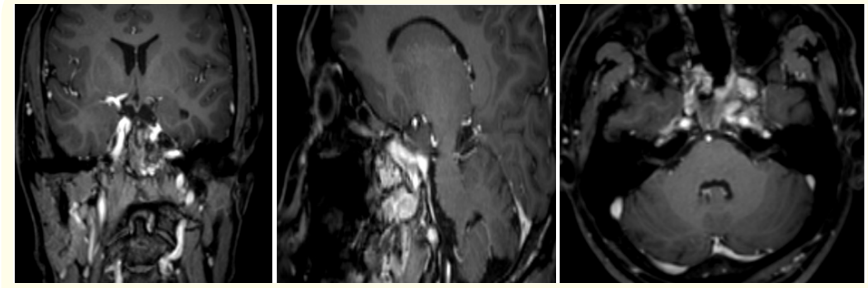

Figure 4a-4c: Shows significant reduction in tumor burden post chemotherapy as demonstrated by the MRI contrast images in coronal, sagittal and axial windows.

The right cavernous sinus became completely free but small remnants were seen on the left side. This was dealt with using Cyber Knife directed specifically at the left sided tumor remnants. He is now on regular follow up for the last 2 months and has, shown minimal increase in size and no new symptoms.

\section{Case Report 2}

A 54 year old lady presented with gradually progressive ophthalmoplegia and diplopia associated with severe shooting pain along the left side of the face. She was treated medically for trigeminal neuralgia without any relief. Imaging showed a dumbbell tumor 
extending across the petrous apex from the posterior fossa to the middle cranial fossa along the cavernous sinus extending upto the orbital apex (Figure 5).

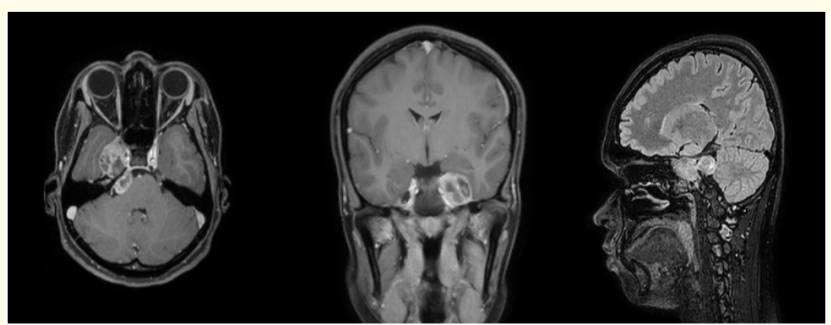

Figure 5: Shows a dumbbell shaped tumor extending from the posterior fossa across the petrous apex to the orbital apex along the cavernous sinus and meddle cranial fossa floor.

Due to the presentation and the radiological features, a diagnosis of Trigeminal Schwannoma was made, and she was posted for surgery. An Orbitozygomatic Approach was made to the middle cranial fossa mass, which was the larger of the two masses. The tumor was seen enclosed in the trigeminal sheath, around the cavernous sinus. Decompression was followed into Meckel's cave, which triggered a trigemino-cardiac reflex which caused the procedure to be abandoned due to severe bradycardia.

Histopathological assessment showed a cellular tumor composed of monomorphic cells in sheets and papillary pattern with intervening proliferation of vasculature. Cells have fine chromatin, moderate to scant cytoplasm with focal fragments showing cells with perinuclear halo are also seen. Multinucleated giant cells also seen in abundance. Areas of haemorrhage, hyalinisation, inflammation, and calcification seen. Possible differentials ranged from Cellular Ependymoma, Oligodendroglioma and atypical GCT were considered. Further Immunohistochemical analysis was planned and the patient was to be started on chemotherapy. She was however lost to follow up.

\section{Discussion}

Giant cell tumors occur in young adults and involves the epiphysio-metaphyseal region of long bones [3-6]. Only a minority of patients present with tumors in axial sites $[7,8]$.

To the best of our knowledge only 16 (including our case) paediatric patients (aged between 7 weeks and 17 years) with GCTs of the cervical spine and 20 children and adolescents with GCTs of the skull base have been reported [4-7].

Most of them were treated with surgery and/or radiotherapy. One patient was reported to have been treated with chemotherapy.
Many of these patients were treated in the pre-RANKL inhibitors era (i.e. in the last century). The first and the only report describing beneficial effects of denosumab in the treatment of the skull base GCT was published recently by Inoue and colleagues, describing a 16 years-old male with a relapsed GCT of the skull base treated with denosumab after failure of the surgery, resulting in marked reduction in tumor size [6]. To the best of our knowledge, our patient is the youngest patient ever reported with a large progressive GCT affecting the skull base and cervical spine, who responded well to therapy with denosumab. As in most patients with spine and skull base GCTs, total surgical excision (which is the standard therapy for GCT) was not feasible as in our patient.

The first patient should have ideally received RT (Radiation Therapy) for his remnant tumor but due to repeated recurrence and poor patient compliance we decided to implement palliative treatment with denosumab [5-8]. Initial cycles of treatment, however, showed excellent improvement in performance status; and MRI performed after $4^{\text {th }}$ treatment cycles confirmed tumor regression.

As complete surgical remission is crucial for long-term survival in patients with GCTs, the long-term prognosis in our patient is expected to be unfavourable. However, as he is tolerating the denosumab therapy without any severe adverse effects, we will continue denosumab. As the second patient was lost to follow up, further adjuvant therapy couldn't be started or monitored.

\section{Conclusion}

GCTs of the skull base are great mimics. They respond to the constraints of space and pressure in the skull base and thus present in a myriad of ways. Proper identification and early surgical excision are important prognostigatory factors in deciding long term outcomes.

Surgery is the treatment of choice for GCTs. In the event of incomplete excision due to location and extent, adjuvant therapy is considered. Radiation is the first line of therapy used but may induce malignant transformation in GCTs. This however is rare and there exists a significant time lag between RT and malignant growth. The second option is chemotherapy. Later advances are agreeable to ensure disease free survival and symptom free survival for variable lengths of time.

\section{Conflict of Interest}

No conflict of Interest exists for the authors.

\section{Funding Support}

No financial grants were received for the publication of this manuscript. 


\section{Bibliography}

1. Fletcher CDM., et al. "Pathology and Genetics of Tumours of Soft Tissue and Bone" (2002).

2. Werner M. "Giant cell tumour of bone: morphological, biological and histogenetical aspects". International Orthopaedics 30.6 (2006): 484-489.

3. Karras NA., et al. "Denosumab treatment of metastatic giantcell tumor of bone in a 10-year-Old girl". Journal of Clinical Oncology 31.12 (2013): e200-e202.

4. Thomas D and Skubitz K. "Giant cell tumour of bone". Current Opinion in Oncology 21.4 (2009): 338-344.

5. Miller IJ., et al. "A case of recurrent giant cell tumor of bone with malignant transformation and benign pulmonary metastases". Diagnostic Pathology 5.1 (2010): 62.

6. Purohit S and Pardiwala DN. "Imaging of giant cell tumor of bone”. Indian Journal of Orthopaedics 41.2 (2007): 91-96.

7. Zhao J., et al. "Giant cell tumor of the clivus: A case report and review of the literature". Oncology Letters 8.6 (2014): 27822786.

8. Chatterjee D., et al. "Sphenoid bone: a rare site for giant cell tumor - case report with literature review". Clinical Neuropathology 35.6 (2016): 385-388.

\section{Assets from publication with us}

- Prompt Acknowledgement after receiving the article

- Thorough Double blinded peer review

- Rapid Publication

- Issue of Publication Certificate

- High visibility of your Published work

Website: www.actascientific.com/

Submit Article: www.actascientific.com/submission.php Email us: editor@actascientific.com

Contact us: +919182824667 